\title{
RESULTS OF THE THORACOSCOPIC RADIOFREQUENCY EPICARDIAL ABLATION WITH A BIPOLAR ELECTRODE DURING LONGSTANDING PERSISTENT FORM OF ATRIAL FIBRILLATION
}

\author{
Rustem Tuleutaev ${ }^{1}$, Alibek Oshakbayev ${ }^{1}$, Kuat Abzaliyev $^{2,3}$, Baurzhan Rakishev ${ }^{1}$, Symbat \\ Abzaliyeva $^{3, *}$ \\ ${ }^{1}$ A.N. Syzgynov National scientific Center of Surgery, 62, Zheltoksan, Almaty, Kazakhstan \\ ${ }^{2}$ Research Institute of Cardiology and Internal Diseases, 120, Aiteke bi, Almaty, Kazakhstan \\ ${ }^{3}$ al-Farabi Kazakh National University, 71 al-Farabi Ave., 050040 Almaty, Republic of Kazakhstan,
}

\begin{abstract}
Annotation Thoracoscopic ablation using the 'box lesion' technique was performed using a bipolar radio frequency clamp. A total of 48 patients, including 38 men and 10 women, mean age 58 years (range $33-74$ ). The mean duration of $\mathrm{AF}$ was 4 yrs (range 1.5 months - 21), the mean size of the atrium $4.15 \pm 0.9 \mathrm{~cm}(2.9-8.8 \mathrm{~cm})$, mean LVEF was $57.7 \%(39-73 \%)$. Mitral regurgitation of 1-2 degrees was present in 14 patients, EDV LV $147.7 \mathrm{ml}$ (81-224). Primary catheter ablation was performed in 22 patients, where 5 of them $(22,7 \%)$ were performed repeatedly. Resection of the left atrial appendage (LAA) during the operation was performed in 44 patients (91\%). Input and output block was achieved in all patients. In the postoperative period, all patients were administered supporting antiarrhythmic therapy with amiodarone and $\beta$-blockers, anticoagulant therapy with warfarin or PLA for 6-12 months. The effectiveness of treatment was monitored by a cardiomonitor Reveal XT in the period 1, 3, $6,12,24$ months after surgery, the mean follow-up length was $498 \pm 19$ days. Sinus rhythm was restored during surgery in all patients and remained until discharge.
\end{abstract}

\section{Introduction}

$\mathrm{AF}$ is the most common form of supraventricular arrhythmia and is characterized by irregular atrial activation without a discrete P wave during ECG [1]. Episode of AF - atrial fibrillation documented on an ECG with a duration of 30 seconds or more (sinus rhythm should be fixed between episodes of AF). Atrial fibrillation (AF) is the most common form of arrhythmia, in which there is a high prevalence and mortality. It was established that pulmonary veins (PV) are the main source of AF development [1]. Catheter radiofrequency ablation aimed at

*Corresponding author: abzaliyeva.symbat@gmail.com 
electrically isolating a drug is effective in the paroxysmal form of AF, however, with this technique, a high relapse rate with persistent form of $\mathrm{AF}$ is noted [2]. Remodelling of the conductive and structural features of the left atrium, which occurs after prolonged episodes of AF maintains AF substrate [3].

The extensive Cox-Maze surgical procedure, which combines isolation of the PV, LAA resection, coagulation of the ligament of Marshall was very effective in the treatment of persistent and long-term persistent forms of AF (duration> 1 year) [4].

Recent studies in contrast-enhanced MRI (Gadolinium) have shown that LA fibrosis is found in areas of low voltage LA in most cases and is an important component of the structural remodeling of AF, which is significantly expressed on the posterior wall of the LA [5]. Low-voltage regions show a reduced conduction velocity and an effective refractory period, which leads to the formation of "reentry" pathways and the preservation of arrhythmia [6]. Previous studies have shown that the presence of zones with low voltage is a predictor of the inefficiency of catheter ablation of AF and the development of recurrence of AF in the future [5]. Accordingly, it was found that the creation of zones on the posterior wall of the LP with the capture of the mouths of the pulmonary veins by the "box lesion" technique reduces the recurrence of AF after ablation in patients with a paroxysmal and (prolonged) persistent form of AF [7]. An operation using unipolar radiofrequency ablation aimed at isolation using the 'box lesion' technique has shown unsatisfactory results, probably due to the insufficiency of the transmural effect [7].

The purpose of this study was to assess the possibility of bipolar thoracoscopic epicardial ablation with isolation of the mouths of the pulmonary veins, confirmed by a bidirectional conduction block in patients with a long-term persistent form of AF.

\section{Materials and methods}

The prospective study group included 48 patients who underwent isolated thoracoscopic RFA of the posterior wall of the LA using the "box lesion" technique, with clipping and resection of the LAA, coagulation of the Marshall ligament and intraoperative testing of the conduction block, from 2016 to July 2019. Inclusion criteria were: patients with a symptomatic longstanding persisting form of AF resistant to antiarrhythmic drugs or traditional catheter RFA, with at least one or three classes of antiarrhythmic drugs being ineffective, long-term persistent AF on ECG before surgery was recorded. The definition of a persistent form of AF was based on the recommendations of the Heart Rhythm Society/European Heart Rhythm Association. Clinical and surgical data were collected in the specialized department of JSC "A.N. Syzganova NSCS" and analyzed. The size of the left atrium was measured by transthoracic echocardiography. Transesophageal echocardiography or MSCT was performed to exclude the thrombus of the LA.

The surgery was performed under general anesthesia using double-lumen endotracheal intubation. Bilateral thoracoscopic access. Two guiding catheters were inserted through oblique and transverse sinuses [8]. An RFA bipolar clamp (Cardioblate Gemini-s, Medtronic Inc.) was attached to the guide catheters and placed so as to enclose the left pulmonary veins and the posterior wall of the LA (Fig. 1). Ablation was performed 5 times with the convexity of the clamp in the direction of the LA and 5 times with the convexity of the clamp in the opposite direction to the posterior wall of the pericardium. Each application continued until a transmural effect was achieved by RFA. This procedure is repeated on the left side, creating a zone of the type "box", which isolates the posterior wall of the LA with the capture of the mouths of the pulmonary veins. Marshall's ligament is dissected. The left atrial appendage (LAA) is isolated using the $60 \mathrm{~mm}$ stapler (Endo GIA Universal Stapler, Covidien Surgical Solutions). Later, an electric cardioversion, if during the ablation it was not possible to restore the sinus rhythm was performed. 


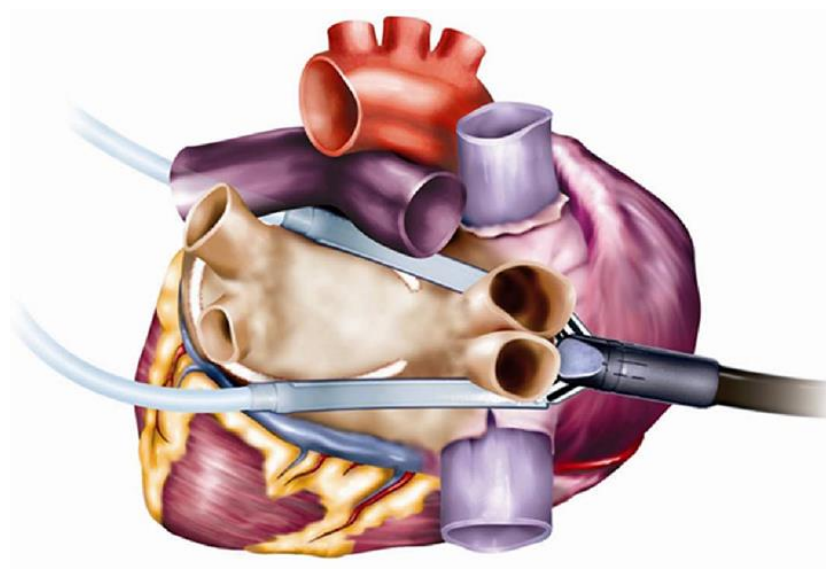

Fig. 1. Creation of the box, posterior wall of the left atrium and pulmonary veins (Medtronic Inc., Minneapolis).

Electrophysiological assessment was carried out by definition Input block: Target - no signals in an isolated area; Output block: The goal was the absence of pulses during stimulation of the heart from an isolated zone. After identifying the mouths of the left pulmonary veins, bipolar electrograms of the left pulmonary veins and the posterior wall of the LA were registered at maximum speed $(0.05 \mathrm{mV} / \mathrm{mm}$ using the MAPS device (Medtronic Inc.). The input unit was confirmed on the basis of the absence of acute electrograms within the pulmonary veins and "box." In the case of restoration of the sinus rhythm, $10.0 \mathrm{~V}$ bipolar stimulation was performed with a pulse width of $2 \mathrm{~ms}$ from the mouths of the pulmonary veins and the "box" zone to ensure the presence of an output block.

The result of ablation was followed up after 1, 3, 6, 12 and 24 months after surgery in accordance with the accepted clinical protocol of treatment. The evaluation was performed using a Reveal XT cardiomonitor and transthoracic Echocardiography (TTE) 3, 6, 12 and 24 months after surgery to evaluate the rhythm or episodes of $\mathrm{AF}$ and the size and function of the LA.

All patients begun or continued to take antiarrhythmic drugs, at least until 6-12 months after surgery, if the patient maintained a sinus rhythm. The administration of oral anticoagulants was resumed and continued for at least 6 months after surgery. Anticoagulants were canceled after 6-12 months at the discretion of the cardiologist and on the basis of the CHADS2VA2SC scale, the presence or absence of successful removal of the LAA and the preservation of the sinus rhythm and contractile function of the LA according to echocardiography. Heart rate was recorded at each observation stage using a Reveal cardiomonitor. The mean follow-up was 498 days. AF recurrence was established when any types of atrial arrhythmias were detected on the electrocardiogram or atrial arrhythmias with a duration of more than $30 \mathrm{~s}$ after 3 months of light period. Patients were asked to visit the clinic in case of symptoms of arrhythmia. Absolute contraindications were: the presence of sinus node weakness syndrome, left atrial appendage thrombosis, the presence of adhesions in the pericardial cavity and pleural cavities after previous operations, moderate and severe COPD, grade 3 obesity.

\section{Results}

Of the 48 patients included in the study, $79 \%$ were men. The mean age was 58 years (33-74 yr), the mean AF duration was $4 y$ (1.5 months to 21 years), and the mean size of the LA was 
$4.15 \pm 0.9 \mathrm{~cm}(2.9-8.8 \mathrm{~cm})$, the mean LVEF was $57.7 \%$ (39-73\%), mitral regurgitation of 12 degrees was present in 14 patients, LV EDV of $147.7 \mathrm{ml}$ (81-224). The most common disease was arterial hypertension which occurred in 41 patients $(85.4 \%)$, coronary heart disease was the least in prevalence: in 5 patients (10.4\%) and diabetes mellitus in 4 patients $(8.3 \%) .4$ patients had a history of cerebrovascular accident. (Table 1 )

Table 1. Main characteristics of the studied patients.

\begin{tabular}{|l|c|c|}
\hline \multicolumn{1}{|c|}{ Characteristics } & $\mathrm{n}$ & $\%$ \\
\hline Men & 38 & 79 \\
\hline Age at the time of the intervention (yr) & 58 & $33-74 \mathrm{y}$ \\
\hline Body mass index (kg/m ${ }^{2}$ ) & 29,7 & \\
\hline Left atrium size (cm) & $4,15 \pm 0,9$ & \\
\hline AF life-duration (years) & $4 \mathrm{y}$ & 1,5 months - 21 years \\
\hline Previous ablation, \% (n) & 22 & 46 \\
\hline Mitral insufficiency 1-2 degree (n) & 14 & 29 \\
\hline Ischaemic heart disease \% (n) & 5 & 10,4 \\
\hline Arterial hypertension \% (n) & 41 & 85,4 \\
\hline Diabetes mellitus \% (n) & 4 & 8,3 \\
\hline Cerebral circulation lesions \% (n) & 4 & 8,3 \\
\hline
\end{tabular}

In the study group, arterial hypertension was detected in $85.4 \%$ of patients, signs of mitral insufficiency were found in $29 \%$, coronary heart disease (CHD) was detected in $10.4 \%, 8.3 \%$ of patients suffered from diabetes, $8.3 \%$ there was a history of acute cerebrovascular accident (Figure 2).

12 months. The effectiveness of treatment was monitored by cardiomonitor Reveal XT in the period 1, 3, 6, 12, 24 months after surgery; the mean follow-up was $498 \pm 19$ days.

Over the entire observation period, 2 deaths occurred: in one patient, while taking warfarin, massive bleeding developed after 10 months; in the second patient, against the background of a stable sinus rhythm, without episodes of AF, after 7 months, according to REAVEL, bradycardia developed with a transition to asystole. In all patients after the intervention, a restoration of the sinus rhythm was observed, which remained until discharge.

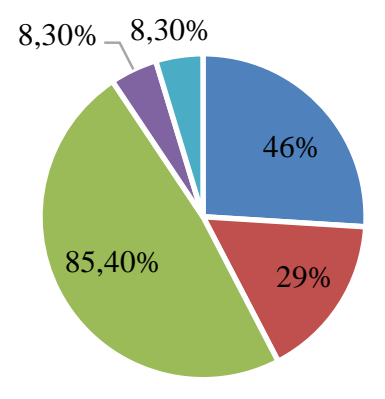

- Mitral insufficiency 1-2 degree - Ischaemic heart disease

- Arterial hypertension - Diabetes mellitus

- Cerebral circulation lesions

Fig. 2. Concomitant diseases. 
Primary catheter ablation was performed in 22 patients ( 5 of them were performed repeatedly). Resection of the LAA during the operation was performed in 44 patients. The input and output block was achieved in all patients.

Table 2. Characteristic of the procedure.

\begin{tabular}{|l|l|}
\hline Ablation time $(\min )$ & 10,46 \\
\hline Operation time $(\min )$ & 183 \\
\hline Cardioversion \% (n) & $72,3 \%(39)$ \\
\hline LAA resection and clipping \% (n) & $92 \%(44)$ \\
\hline
\end{tabular}

All patients were discharged with sinus rhythm. In 1 patient, pneumothorax was noted and therefore left-side thoracotomy was performed in the postoperative period. In 1 patient, a conversion was performed due to bleeding. It was also noted that during ablation, insufficient transmurality was visually observed in the part where the base is cauterized by a bipolar clamp, so in one patient after surgery, an impulse break is observed during the mapping of this zone.

Complications during the procedure were observed in $4 \%$ of the subjects. Pneumothorax was observed in 1 patient, and therefore left-side thoracotomy was performed in the postoperative period, and 1 patient showed sinus node weakness syndrome after the procedure, for which a temporary pacemaker was installed. In two cases, a minithoracotomy was performed due to the adhesion process of the lungs. In these cases, the procedures were performed through minimally invasive thoracotomy instead of thoracoscopic access. During this procedure, sections providing access to the chest were $6-7 \mathrm{~cm}$ long. This increased the invasiveness of the procedure, which probably led to higher risks of complications.

In the postoperative period all patients were receiving supporting antiarrhythmic therapy with amiodarone and $\beta$-blockers, anticoagulant therapy with warfarin or PLA for 6-

\section{Discussion}

The study evaluates the effectiveness of thoracoscopic isolation of the mouths of the pulmonary veins according to the "box lesion" method with intraoperative measurement of the conduction block degree in the treatment of a longstanding persisting form of AF.

The main findings of this study are:

- High efficiency of isolation of the mouths of the pulmonary veins according to the "box lesion" method using a bipolar electrode in the treatment of a longstanding persisting form of AF;

- High reliability of intraoperative testing of isolation of the mouths of the pulmonary veins according to the "box lesion" method using electrostimulating devices and an implantable Reavel XT cardiomonitor.

Currently, there are studies confirming that thoracoscopic drug ablation is a feasible and safe procedure [13]. However, there is evidence that thoracoscopic isolation according to the "box lesion" technique, performed using a monopolar electrode, does not lead to isolation according to the "box lesion" type and long-term preservation of the sinus rhythm [8]. As a result of the use of monopolar ablation, it is not possible to achieve a transmural damaging effect, which does not contribute to a long-term antiarrhythmic effect [14]. Incomplete damaging effects are a risk factor for recurrence of $\mathrm{AF}$ and the induction of other forms of arrhythmia of the LA [15]. Therefore, ablation using a bipolar energy source is the preferred technique, since the energy is directed to the entire thickness of the LA walls between the jaws of the clamp, increasing the likelihood of a transmural effect of the energy used. In addition, intraoperative testing of the impulse conduction block during this procedure is quite 
possible and can confirm the transmural damaging effect. This effect was confirmed by Dr. Krul and others who performed minimally invasive hybrid surgical ablation under electrophysiological control in a group of patients with paroxysmal AF and in a group of patients with (long-term) persistent AF [16].

Patients with a prolonged persistent form of $\mathrm{AF}(\mathrm{n}=48)$ were isolated from the pulmonary veins with additional ablation of the LA until a bidirectional block was confirmed. This procedure was possible and showed a high frequency of successful results for the entire observation period. The average duration of ablation was 10 min $46 \mathrm{sec}$. In 9 patients (18.7\%), the sinus rhythm was restored spontaneously during ablation, and in 39 patients $(72.3 \%)$ after electrical cardioversion. Sinus rhythm was maintained during the year of observation after surgery in $87.5 \%$ of patients.

Our study showed that performing thoracoscopic epicardial ablation according to the "box lesion" method using bipolar RFA showed high effectiveness in the prevention of relapses both in the early period after surgery and in the long-term follow-up ( 24 months) of observation.

Sinus rhythm in all 48 patients was restored during surgery and remained until discharge. It was also noted that during ablation, insufficient transmurality was visually observed in the part where the base is cauterized by a bipolar clamp, so in one patient after surgery, an impulse break is observed during the mapping of this zone. In the postoperative period after 30 days in $2 \%$ of patients against dilatation of the cardiac cavities and low LVEF $(36 \%)$ flutter of the right atrium was noted, in $4.1 \%$ - flutter of the left atrium was noted in the 2nd year (for which a successful transcatheter RFA was performed).

In the observation group, in $12.5 \%$ of patients, a relapse of AF occurred in the observation period from 30 days to 2 years, in $6 \%$ of patients with a sinus rhythm short episodes (no more than 30 seconds) of AF were observed a year after the operation. Thus, a good effect on AF prevention was achieved in $87.5 \%$ of patients after thoracoscopic radiofrequency ablation of pulmonary veins using the "box lesion" technique using a bipolar electrode and intraoperative block testing.

\section{Conclusion}

Thoracoscopic radiofrequency ablation of pulmonary veins using the "box lesion" technique using a bipolar electrode and intraoperative conduction block testing is a promising treatment for atrial fibrillation, which is accompanied by a high efficiency of $87.5 \%$, a low risk of complications (4\%), especially with catheter ablation and in patients with large sizes of the left atrium. The combination of this method of treatment with catheter ablation of the left or / and right isthmus gives greater efficiency.

\section{References}

1. V. Fuster, L. E. Rydén, C annom DS, H. J. Crijns, A. B. Curtis, K. A. Ellenbogen et al. European Society of Cardiology Committee for Practice Guidelines; European Heart Rhythm Association; (Heart Rhythm SocietyACC/AHA/ESC 2006)

2. Guidelines for the Management of Patients with Atrial Fibrillation: a report of the American College of Cardiology/American Heart Association Task Force on Practice Guidelines and the European Society of Cardiology Committee for Practice Guidelines (Writing Committee to Revise the 2001)

3. Guidelines for the Management of Patients With Atrial Fibrillation): developed in collaboration with the European Heart Rhythm Association and the Heart Rhythm Society, Circulation , 114 (257-354) (2006) 
4. M. Haissaguerre, P. Jais, D.C. Shah et al, N Engl J Med, Spontaneous initiation of atrial fibrillation by ectopic beats originating in the pulmonary veins. 339 (59-66) 1998

5. A. W. Teh, P.M. Kistler, G. Lee et al, J Cardiovasc Electrophysiol, Electroanatomic remodeling of the left atrium in paroxysmal and persistent atrial fibrillation patients without structural heart disease. 23 (232-8) (2012)

6. M. La Meir, S. Gelsomino, F. Lucà, R. Lorusso, G.F. Gensini, L. Pison, F. Wellens, J. Maessen, Minimally invasive thoracoscopic hybrid treatment of lone atrial fibrillation: early results of monopolar versus bipolar radiofrequency source. Interactive CardioVascular and Thoracic Surgery, Volume 14, Issue 4, April 2012, Pages 445-450;

7. F. Fochler, T. Yamaguchi, M. Kheirkahan, E.G. Kholmovski, A.K. Morris, N.F. Marrouche, Late Gadolinium Enhancement Magnetic Resonance Imaging Guided Treatment of Post-Atrial Fibrillation Ablation Recurrent Arrhythmia. Circulation: 12007174. https://doi.org/10.1161/CIRCEP.119.007174; (Arrhythmia and Electrophysiology. 2019)

8. J. L. Cox, J Thorac, Cardiovasc Surg, Atrial fibrillation I: a new classification system, 126 (1686-92) (2003)

9. H. Oral, B.P. Knight, H. Tada et al. Pulmonary vein isolation for paroxysmal and persistent atrial fibrillation. Circulation. 105(1077-81) (2002)

10. T. Sueda, N. Uchida, T. Takasaki et al. Long-term results after the box pulmonary vein isolation procedure for chronic atrial fibrillation in mitralvalve surgery, 18 (101-8) (Ann Thorac Cardiovasc Surg. 2012)

11. R. Cappato, H. Calkins, S.A. Chen, W. Davies, Y. Iesaka, J. Kalman, Y.H. Kim, G. Klein, D. Packer, A. Skanes. Worldwide Survey on the Methods, Efficacy, and Safety of Catheter Ablation for Human Atrial Fibrillation. Circulation. 2005;111:1100-1105. 21 Feb 2005;

12. P Gal, J. J. Smit, A. Adiyaman, A. R. Ramdat Misier, P. P Delnoy, A. Elvan First Dutch, Neth Heart J experience with the endoscopic laser balloon ablation system for the treatment of atrial fibrillation, 23 (96-9) (2015)

13. L. J. Vries de, F. Akca, M. Khan et al. Clinical outcome of ablation for long-standing persistent atrial fibrillation with or without defragmentation. Neth Heart J. 22 (30-6) (2014)

14. K. Kumagai, S. Muraoka, C. Mitsutake, H. Takashima, H. Nakashima. A new approach for complete isolation of the posterior left atrium including pulmonary veins for atrial fibrillation. J Cardiovasc Electrophysiol. 18 (1047-52) (2007)

15. J. C. Pruitt, R.R. Lazzara, G. Ebra, Minimally invasive surgical ablation of atrial fibrillation: the thoracoscopic box lesion approach. J Interv Card Electrophysio 120 (83-7) (2007)

16. Y. Miyagi, Y. Ishii, T. Nitta, M.Ochi, K. Shimizu. Electrophysiological and histological assessment of transmurality after epicardial ablation using unipolar radiofrequency energy. J Card Surg. 24 (34-40) (2009)

17. S.P .Thomas, T. W Lim, McCall R, S.C. Seow, D. L Ross, Electrical isolation of the posterior left atrial wall and pulmonary veins for atrial fibrillation: feasibility of and rationale for a single-ring approach. Heart Rhythm, (4)722-30 (2007)

18. S. P Krul, L. Pison, La Meir M, et al. Epicardial and endocardial electrophysiological guided thoracoscopic surgery for atrial fibrillation: a multidisciplinary approach of atrial fibrillation ablation in challenging patients. Int J Cardiol, 173 (229-3) ( 2014) 